\title{
Minimum-time rest-to-rest feedforward action for PID feedback MIMO systems
}

\author{
Luca Consolini* Gabriele Lini* Aurelio Piazzi* \\ Antonio Visioli ${ }^{* *}$ \\ * Dipartimento di Ingegneria dell'Informazione, University of Parma, \\ Italy, \{luca.consolini, aurelio.piazzi\}@unipr.it, gabriele.lini@gmail.com \\ ** Dipartimento di Ingegneria dell'Informazione, University of Brescia, \\ Italy, antonio.visioli@ing.unibs.it
}

\begin{abstract}
In this paper we present a methodology for the design of a feedforward control law to be applied to a closed-loop PID-based control system for a multi-input multi-output process in order to achieve a minimum-time rest-to-rest transition of the system from an equilibrium point to another subject to constraints on both the control and process variables. In particular, the proposed approach uses decentralized PID controllers which can be designed by any of the conventional methods, such as, for example, those aiming at rejection of load disturbances. Then, the closed-loop generalized bang-bang command input vector is determined by applying a linear programming approach in order to minimize the rest-to-rest output transitions. Conditions for the constraints for which the problem admits a solution are given. Simulations for a two-inputs two-outputs plant highlight the effectiveness of the approach.
\end{abstract}

Keywords: minimum-time control, constrained control, feedforward control

\section{INTRODUCTION}

Proportional-Integral-Derivative (PID) controllers are the most employed controllers in industry because of their relative ease of use and because they can provide a satisfactory performance for the vast majority of industrial processes. In order to simplify their use, many tuning rules have been devised in the context of both single-input-single-output (SISO) [1] and multiple-input-multiple-output (MIMO) systems (see for example $([2,3]))$.

Indeed, the success of PID controllers is also due to the fact that when a tight performance is required, the PID-based control scheme can be improved effectively by employing additional functionalities (such as set-point weight, antiwindup, decoupling, feedforward action) that make the control system capable to satisfy the control specifications by keeping at the same time the overall control design simpler than using methods based on advanced control theory $[4,5]$.

One of these additional functionalities is the feedforward control action, which can be suitably applied in order to improve the set-point following performance, especially when the tuning of the PID parameters is devoted to the load disturbance rejection performance. Actually, different techniques have been devised for the synthesis of a feedforward action in the context of SISO systems, exploiting different concepts such as set-point weight $[6,7]$, bangbang control $[8,9]$, and input-output inversion [10]. Some of these techniques have been also extended to MIMO processes $[11,12]$. In this context, it is important to take into account that, in order to achieve a high performance in practical applications, constraints on both the the system inputs and outputs should be considered explicitly in the design phase ([13]). Indeed, there are always saturation limits for the actuators and many times the process variables cannot exceed given limit values in order to satisfy the control specifications.

Extending to the MIMO case the results presented in [14], in this paper we propose the application of a minimum- time feedforward control strategy to a closed-loop MIMO system with PID controllers where both actuator limits as well as constraints on the maximum overshoot and undershoot of the outputs are taken into account. In other words, we determine the command inputs to be applied to the closed-loop system in order to provide a minimumtime rest-to-rest transition from an equilibrium state to another (corresponding to desired transitions of the process outputs from a set-point value to another) subject to minimum and maximum constraints for the manipulated variables as well as for the process variables.

The solution is based on a linear programming approach. Specifically, a discretization of the continuous-time MIMO plant is first computed. Then, a sequence of linear programming feasibility tests leads to the determination of the time-optimal feedforward action. It is worth noting that, with respect to the solution based on Chebyshev polynomials proposed in [12], here there is no series approximation of the dynamics of the system and of the feedforward action.

The paper is organized as follows. Section 2 introduces the set $\mathcal{T}_{\mathrm{p}}$ of all rest-to-rest transitions for a given multivariable linear plant and an inclusion condition for which $\mathcal{T}_{\mathbf{p}}$ is not empty is given in Theorem 1. Then, the minimumtime rest-to-rest transition problem can be posed (cf. (12)). Subsections 2.2 and 2.3 describe the discretization method and the linear programming approach to solve the posed time-optimal problem. Results are presented in Section 3 where simulations for two examples are reported. Concluding remarks end the paper in Section 4.

Notation: for a vector $u \in \mathbb{R}^{m},\|u\|=\sqrt{u^{T} u}$ denotes its 2norm; given a function $u: t \rightarrow \mathbb{R}^{m},\|u\|_{\infty}=\sup _{t \in \mathbb{R}}\|u(t)\|$ denotes the infinity norm. For a square matrix $M,\|M\|$ denotes the matrix 2 -norm. For a $n$-degree polynomial ma$\operatorname{trix} M(s)=M_{0}+M_{1} s+\ldots M_{n} s^{n},\|M(s)\|=\sum_{i=0}^{n}\left\|M_{i}\right\|$. For a $C^{n}$ function $u,\|u\|_{\infty, n}=\sum_{i=0}^{n}\left\|\frac{d^{i}}{d t^{i}} u\right\|_{\infty}$. Notation $M\left(\frac{d}{d t}\right)$ represents the linear operator obtained by sub- 


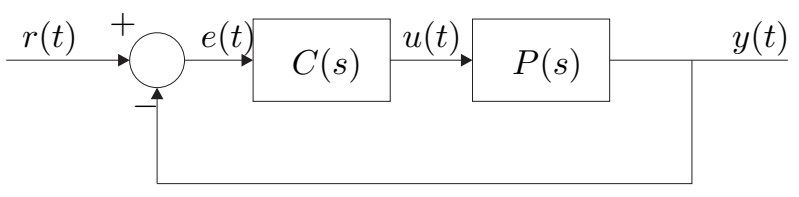

Fig. 1. The control scheme.

stituting the variable of polynomial matrix $M$ with the derivation. A consequence of these definitions is that for any $C^{n}$ function $u$ and any $n$-degree matrix polynomial $M(s),\left\|M\left(\frac{d}{d t}\right) u\right\|_{\infty} \leq\|M(s)\|\|u\|_{\infty, n}$. Given a set $U \subset \mathbb{R}^{n}$, int $(U)$ represents its interior. Function $1(t)$ is the unit step function.

\section{THE MINIMUM-TIME FEEDFORWARD CONSTRAINED REGULATION PROBLEM}

The MIMO feedback control scheme considered in this work is presented in Figure 1. The set point vector function $r$ is assumed to be a function of time and the problem consists in finding the optimal set-point vector signal $r$ such that the output vector $y$ performs a rest-to-rest transition in minimum time and the plant $P$ reaches the equilibrium at the end of the transition time (i.e. both the system input and output are constant).

In this control scheme, plant $P(s)$ may be unstable and is stabilized by controller $C(s)$. In this way, the transfer functions from the set-point signal $r$ to outputs $u$ and $y$ are asymptotically stable. The problem is equivalent to the one of designing an (unconstrained) set-point signal $r$ so that the resulting signals $u$ and $y$ obey specified input and output constraints. In the following, to solve this problem, we extend to the multivariable case some of the methods presented in [15] for stable SISO systems. Nevertheless, they can be also applied to unstable plants $P(s)$ connected with a stabilizing controller $C(s)$ as in figure 2 , since the transfer function from input $r$ to outputs $u$ and $y$ is stable.

\subsection{Feedforward constrained minimum-time regulations}

Consider a linear, stable, continuous-time MIMO system $\Sigma$ with $m$ inputs and $p$ outputs, described by the strictly proper transfer function matrix $H(s)=N(s) D^{-1}(s) \in$ $\mathbb{R}^{m \times p}$, where $D(s)$ and $N(s)$ are polynomials matrices.

The system static gain matrix is $H(0)$ and the input and output vectors are denoted by $u$ and $y$ respectively. With $h(t)$ we denote the impulse response matrix of system $\Sigma$, i.e. $h(t)=\mathcal{L}^{-1}[H(s)]$ where $\mathcal{L}^{-1}$ denotes the inverse Laplace transform.

The behavior set of $\Sigma$ can be introduced as the set $\mathcal{B}$ of all input-output pairs $(u, y) \in L_{1}^{\text {loc }} \times \in L_{1}^{\text {loc }}$ that are "weak" solutions of the differential equation [16]:

$$
D\left(\frac{d}{d t}\right) y=N\left(\frac{d}{d t}\right) u \text {. }
$$

Let $y_{0}$ and $y_{1}$ be desired initial and final rest values for the system output and $u_{0}$ and $u_{1}$ corresponding values for the input (i.e. $y_{0}=H(0) u_{0}$ and $y_{1}=H(0) u_{1}$ ).

The control aim is to find a minimum-time feedforward input that causes a rest-to-rest transition from $y=y_{0}$ to $y=y_{1}$ subject to arbitrarily assigned input and output constraints $\left(y_{0}, y_{1} \in \mathbb{R}^{p}\right.$ are any desired initial and final output values). The rest condition of $\Sigma$ is characterized by the set of input-output equilibrium points designated as
$\mathcal{E}:=\left\{(u, y) \in \mathbb{R}^{m} \times \mathbb{R}^{p}: y=H(0) u\right\}$. We introduce, as a special subset of $\mathcal{B}$, the set $\mathcal{T}_{\mathbf{p}}$ of all rest-to-rest transitions from $\left(u_{0}, y_{0}\right) \in \mathcal{E}$ to $\left(u_{1}, y_{1}\right) \in \mathcal{E}$ subject to input and output constraints.

Definition 1. Let be given a constraint parameter set $\mathbf{p}:=$ $\left\{\mathcal{U}, \mathcal{Y}, y_{0}, y_{1}\right\}$ where $\mathcal{U}$ and $\mathcal{Y}$ are convex sets that represent the constraints for the input and output respectively, while $y_{0}$ and $y_{1}$ are the initial and final output rest values for the output. Let $u_{0}$ and $u_{1}$ be such that $y_{0}=H(0) u_{0}$, $y_{1}=H(1) u_{1}$ and assume

$$
\left\{u_{0}, u_{1}\right\} \subset \mathcal{U} \text { and }\left\{y_{0}, y_{1}\right\} \subset \mathcal{Y} \text {. }
$$

Define $\mathcal{T}_{\mathbf{p}}$ as the set of all pairs $(u, y) \in \mathcal{B}$ for which there exists $t_{f}>0$ such that:

$$
\begin{gathered}
u(t)=u_{0} \quad \forall t<0, \quad u(t)=u_{1} \forall t \geq t_{f}, \\
u(t) \in \mathcal{U} \quad \forall t \in\left[0, t_{f}\right], \\
y(t)=y_{0} \quad \forall t<0, \quad y(t)=y_{1} \quad \forall t \geq t_{f}, \\
y(t) \in \mathcal{Y} \quad \forall t \in\left[0, t_{f}\right] .
\end{gathered}
$$

The constraints intervals introduced in the above definition can encapsulate all the typical amplitude limitations that apply to the input and the output for any set-point regulation problem.

Lemma 1. Let system $\Sigma$ be controllable, then for any $T_{a}>T_{b}$, there exists a positive constant $M$ such that for any couple of input-output pairs $\left(u_{a}, y_{a}\right),\left(u_{b}, y_{b}\right) \in \mathcal{B}$, there exists a pair $(u, y) \in \mathcal{B} \cap C^{n}$ such that

1) $u(t)=u_{a}(t), y(t)=y_{a}(t)$ for $t \leq T_{a}$,

2) $u(t)=u_{b}(t), y(t)=y_{b}(t)$ for $t \geq T_{b}$,

3) $\left\|\left[u-u_{a}, y-y_{a}\right]\right\|_{\infty} \leq M\left\|\left[u_{a}-u_{b}, y_{a}-y_{b}\right] \mid 1\left(t-t_{a}\right)\right\|_{\infty}$.

Sketch of the proof. We proceed along the lines of the proof of Theorem 5.2 .5 of $[16]$. Let $R(s)=[D(s), N(s)]$, then $(u, y) \in \mathcal{B}$ if $R\left(\frac{d}{d t}\right)[u, y]^{T}=0$. Let $n$ be the degree of polynomial matrix $R(s)$. There exists unimodular matrices $U(s), V(s)$ such that $\bar{R}(s)=U(s) R(s) V(s)=[D(s), 0]$ is in Smith form. Let $w_{a}=V^{-1}\left[u_{a}, y_{a}\right]$ and $w_{b}=$ $V^{-1}\left[u_{b}, y_{b}\right]$. There exists an interpolated $C^{n}$ function $w$ such that $w=u_{a}$, for $t \leq t_{a}, w=w_{b}$ for $t \geq t_{b}$ and a constant $N$ such that $\left\|w-w_{a}\right\|_{\infty, n} \leq N \| \bar{w}_{b}-$ $w_{a} \|_{\infty, n}$. The interpolated function can be obtained, for instance, using Hermite interpolation polynomials and the existence of the bounding constant $N$ depends from the properties of Hermite polynomials. The input-output couple $[u, y]=V\left(\frac{d}{d t}\right) w$ satisfies $\|\left[u-u_{a}, y-y_{a}\right] 1(t-$ $\left.t_{a}\right)\left\|_{\infty} \leq\right\| V(s)\|\| w_{b}-w_{a} \|_{\infty, n}$ and satisfies the thesis with $M=N\|V(s)\|_{\infty}\left\|V(s)^{-1}\right\|_{\infty}$.

The following theorem gives a straightforward sufficient condition to ensure that $\mathcal{T}_{\mathbf{p}}$ is not empty.

Theorem 1. Set $\mathcal{T}_{\mathbf{p}}$ is not empty if

$$
\left\{u_{0}, u_{1}\right\} \subset \operatorname{int}(\mathcal{U}) \text { and }\left\{y_{0}, y_{1}\right\} \subset \operatorname{int}(\mathcal{Y}) .
$$

\section{Proof.}

Let $l(t)$ be any $C^{n}$ function such that

$$
\begin{aligned}
& l(t)=u_{0} \forall t<0, l(t)=u_{1} \forall t>1, \\
& u_{0} \leq l(t) \leq u_{1} \forall t \in[0,1],
\end{aligned}
$$

where operator $\leq$ denotes the componentwise inequality.

Given a real constant $\epsilon>0$, let the input to system $\Sigma$ be given by $l(\epsilon t)$ and denote by $y(t ; \epsilon)$ the corresponding 
output with $y(t ; \epsilon)=0 \forall t<0$. Hence, the following limit holds:

$$
\lim _{\epsilon \rightarrow 0}\|y(t ; \epsilon)-l(\epsilon t) H(0)\|_{\infty}=0
$$

Indeed, the Laplace transform of $y(t ; \epsilon)-l(\epsilon t) H(0)$ is given by:

$$
L(s ; \epsilon)(H(s)-H(0)),
$$

where $L(s ; \epsilon):=\mathcal{L}[l(\epsilon t)]$. Since $H(s)-H(0)=s \tilde{H}(s)$, where $\tilde{H}(s)$ is a suitable stable biproper transfer function matrix, expression $(9)$ can be written as $L(s ; \epsilon) s \tilde{H}(s)=$ $\tilde{H}(s) \mathcal{L}\left[\frac{d}{d t} l(t \epsilon)\right]$. Therefore

$$
\|y(t ; \epsilon)-l(\epsilon t) H(0)\|_{\infty} \leq \int_{0}^{+\infty}\|\tilde{h}(v)\| d v \cdot\left\|\frac{d}{d t} l(t \epsilon)\right\|_{\infty},
$$

where $\tilde{h}(t)=\mathcal{L}^{-1}[\tilde{H}(s)]$ and $\int_{0}^{+\infty}\|\tilde{h}(v)\| d v$ is the peak gain of $\tilde{H}(s)$. Since $\left\|\frac{d}{d t} l(t \epsilon)\right\|_{\infty}=\epsilon\left\|\frac{d}{d(t \epsilon)} l(t \epsilon)\right\|_{\infty}$, limit (8) is proved. Moreover, from $\frac{d^{i}}{d t^{2}} l(t \epsilon)=\epsilon^{i} \cdot \frac{d^{i}}{d(t \epsilon)^{2}} l(t \epsilon)$ we have

$$
\lim _{\epsilon \rightarrow 0}\left\|\frac{d^{i}}{d t^{i}} l(t \epsilon)\right\|_{\infty}=0, i=1, \ldots, n-1 .
$$

Then, using again the peak gain concept it follows that

$$
\lim _{\epsilon \rightarrow 0}\left\|\frac{d^{i}}{d t^{i}} y(t ; \epsilon)\right\|_{\infty}=0, i=1, \ldots, n-1 .
$$

So far we have constructed a family of input-output pairs $(l(\epsilon t), y(t ; \epsilon)) \in \mathcal{B}$ parameterized by $\epsilon>0$. Now, apply lemma 1 using $\left(u_{a}, y_{b}\right)=(l(\epsilon t), y(t ; \epsilon))$ and $\left(u_{b}, y_{b}\right)=$ $\left(u_{1}, y_{1}\right)$, setting $T_{a}=\epsilon^{-1}$ and $T_{b}=\epsilon^{-1}+1$. By lemma 1 , since $\left[l(\epsilon t)-u_{1}, y(t ; \epsilon)-y_{1}\right]$ can be made arbitrarily small by reducing $\epsilon$, it follows that there exists a set of pair $(u, y)$ that satisfies the rest-to-rest conditions and such that

$$
\lim _{\epsilon \rightarrow 0}\|(u-l(\epsilon t), y-y(t ; \epsilon))\|_{\infty}=0,
$$

this implies that there exists a sufficiently high value for $\epsilon$ for which the pair $(u, y)$ satisfies the provided bounds.

Remark 1. Note that sufficient condition (7) differs from assumption (2) of Definition (1) defining $\mathcal{T}_{\mathbf{p}}$ just for the exclusion of the boundaries of sets $U_{c}$ and $Y_{c}$. Hence, condition (7) implies that there exists at least a small distance between the constraints extrema and the corresponding steady-state input-output values. This permits to construct (as shown in the proof) an input-output pair that reaches the steady-state in finite time while respecting the constraints.

Once inclusions (7) are satisfied, the emerging natural problem is to determine among all the constrained transitions of $\mathcal{T}_{\mathbf{p}}$ the fastest one, i.e. the optimal rest-to-rest transition with associated minimum transition time $t_{f}^{*}$ :

$$
t_{f}^{*}:=\inf _{(u, y) \in \mathcal{T}_{\mathbf{p}}} T_{f}(u, y)
$$

where $T_{f}$ is the following functional

$$
\begin{gathered}
T_{f}(u, y)=\inf \left\{t_{1}: u(t)=u_{1},\right. \\
\left.y(t)=y_{1}, \forall t \geq t_{1}\right\}
\end{gathered}
$$

which is well defined by Definition 1 . Note that $t_{f}^{*}$ corresponds to the minimum $T_{f}(u, y)$ that is achievable with an optimal pair $\left(u^{*}, y^{*}\right)$.

On the other hand, from a control viewpoint the problem is to directly determine the optimal feedforward input $u^{*}(t)$ that corresponds to minimum-time $t_{f}^{*}$. An approximate solution to this problem using linear programming will be exposed.
Remark 2. Even though the above theorem provides only a sufficient condition it is worth noting that inclusions (7) are almost necessary. Indeed if $u_{0} \notin \mathcal{U} \vee u_{1} \notin \mathcal{U} \vee$ $y_{0} \notin \mathcal{Y} \vee y_{1} \notin \mathcal{Y}$ then $\mathcal{T}_{\mathbf{p}}$ is evidently empty because of the incompatibility of the input-output rest values with the required constraints.

\subsection{Discrete-time minimum-time constrained control synthesis}

In this section, the minimum-time feedforward control problem is approximated with a discrete-time one and a solution is provided using linear programming. A linear discrete-time system $\Sigma_{d}$ is described by the scalar transfer function matrix

$$
P(z)=H(z)=A^{-1}(z) B(z) .
$$

$\Sigma_{d}$ is stable, and its static gain is $P(1)$. The system input and output sequences are denoted by $u(k)$ and $y(k)$ respectively, $k \in \mathbb{Z}$.

The behavior $\mathcal{B}_{d}$ of system $\Sigma_{d}$ is the set of all input-output pairs $(u, y)$, where $u, y: \mathbb{Z} \rightarrow \mathbb{R}$, satisfying the difference equation:

$$
A(d) y=B(d) u
$$

where $d$ represents the 1-step delay operator, such that for any function $u(k), d(u)=u(k-1)$.

The set of input-output equilibrium points of $\Sigma_{d}$ is $\mathcal{E}:=$ $\{(u, y): y=P(1) u\}$ and the set $\mathcal{K}_{\mathbf{p}} \subset \mathcal{B}_{d}$ of all rest-to-rest constrained transitions from $\left(u_{0}, y_{0}\right) \in \mathcal{E}$ to $\left(u_{1}, y_{1}\right) \in \mathcal{E}$ is defined as follows.

Definition 2. Given the parameter set $\mathbf{p}:=\left\{\mathcal{U}, \mathcal{Y}, y_{0}, y_{1}\right\}$ where $\mathcal{U}$ and $\mathcal{Y}$ are convex sets and $y_{0}, y_{1}$ are the initial and final rest value of the output, $\mathcal{K}_{\mathbf{p}}$ is the set of all pairs $(u, y) \in \mathcal{B}_{d}$ for which there exists $k_{f} \in \mathbb{N}$ such that:

$$
\begin{array}{cc}
u(k)=u_{0} \quad \forall k<0, \quad u(k)=u_{1} \quad \forall k \geq k_{f}, \\
u(k) \in \mathcal{U} \quad \forall k \in \mathbb{Z}, \\
y(k)=y_{0} \quad \forall k<0, \quad y(k)=y_{1} \quad \forall k \geq k_{f}, \\
y(k) \in \mathcal{Y} \quad \forall k \in \mathbb{Z} .
\end{array}
$$

The following result is the discrete counterpart of Theorem 1.

Theorem 2. Set $\mathcal{K}_{\mathbf{p}}$ is not empty if

$$
\left\{u_{0}, u_{1}\right\} \subset \operatorname{int}(\mathcal{U}) \text { and }\left\{0, y_{f}\right\} \subset \operatorname{int}(\mathcal{Y}) .
$$

Proof. It is analogous to the proof of Theorem 1. For brevity, it is omitted.

The minimum-time feedforward constrained control problem for discrete-time systems consists in finding the optimal input sequence $u^{*}(k), k=0,1, \ldots, k_{f}^{*}-1$ for which the pair $\left(u^{*}, y^{*}\right) \in \mathcal{K}_{\mathbf{p}}$ is a minimizer for the optimization problem:

$$
k_{f}^{*}=\min _{(u, y) \in \mathcal{K}_{\mathbf{p}}} K_{f}(u, y) .
$$

$K_{f}(u, y)$, the rest-to-rest transition time associated to pair $(u, y)$, is defined as follows

$$
\begin{aligned}
& K_{f}(u, y):= \\
& \min \left\{k_{1} \in \mathbb{N}: u(k)=u_{1}, y(k)=y_{1}, \forall k \geq k_{1}\right\} .
\end{aligned}
$$

Let $T_{k}: \mathbb{R}^{m \times k_{f}} \rightarrow \mathbb{R}^{p \times k_{f}}$ be the linear transformation that associates to the input vector $\left(v(0), v(1), \ldots, v\left(k_{f}-\right.\right.$ 


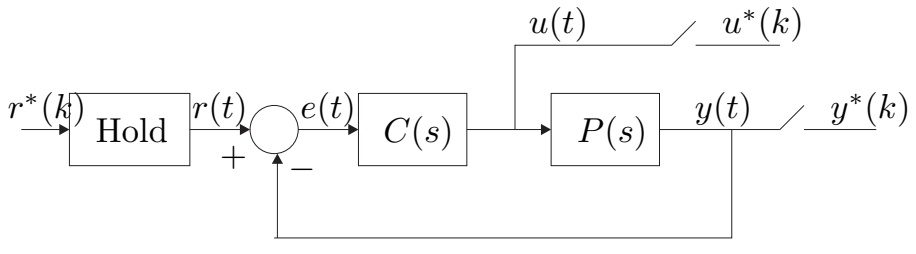

Fig. 2. The discrete control scheme.

1)) the output $y(k)$, where $y$ is the output function that corresponds to the input

$$
\begin{aligned}
& u(k)=u_{0} \text { if } k<0 \\
& u(k)=v(k) \text { if } 0 \leq k<k_{f} \\
& u(k)=u_{1} \text { if } k \geq k_{f} .
\end{aligned}
$$

The following proposition shows that a constrained transition in $k_{f}$ steps exists if and only if a convex programming problem is feasible.

Proposition 1. There exists an input-output couple $(u(k), y(k))$ which is the solution to Problem 1 if and only if there exist $k_{f} \in \mathbb{N}$ and a vector $u \in \mathbb{R}^{k_{f}}$ for which the following convex problem is feasible:

$$
\begin{gathered}
u(k) \in \mathcal{U}, \forall k=0, \ldots, k_{f}-1 \\
T_{k}\left(u(0), u(1), \ldots, u\left(k_{f}-1\right)\right) \in \mathcal{U}, \forall k=0, \ldots, k_{f}-1 \\
T_{k}\left(u(0), u(1), \ldots, u\left(k_{f}-1\right)\right)=y_{f}, \\
\forall k=k_{f}, \ldots, k_{f}+N-1,
\end{gathered}
$$

where $N$ is the degree of transfer function $H$.

In this way, the minimum number of steps $k_{f}^{*}$ and the corresponding optimal control $u^{*}$ can be determined with a bisection algorithm.

Idea of the proof. The result is straightforward, if control $u$ satisfies the proposed convex feasibility problem, then the input and output constraints are satisfied. Moreover, since the input and output signals are constant for $N$ steps from $k_{f}$, they are at rest conditions.

\subsection{Discretization of the constrained control problem}

The continuous control problem can be approximated with a discrete one as follows. Consider the scheme represented in Figure 2 with sampling time $T$. This system corresponds to the one represented in Figure 1, with the difference that the control function is assumed to be given by the piecewise control signal $r(t)$ obtained from the discretetime one $r^{*}(k)$ through a zero-order hold. Signals $u^{*}(k)$ and $y^{*}(k)$ are obtained by sampling the continuous input and output $u(t)$ and $y(t)$.

Problem 1. With reference to the control scheme presented in Figure 2, find a discrete-time reference signal $r(k)$ such that the number of discrete steps $k_{f}^{*}$ required for the transition is minimum, i.e. equation (21) is satisfied.

This problem can be solved with tho different approaches:

- The set-point signal $r^{*}$ is considered as the system (unconstrained) input and the signals $u^{*}$ and $y^{*}$ are considered as two different (constrained) outputs. The optimization procedure is applied directly to the closed-loop transfer function from $r^{*}$ to $u^{*}$ and $y^{*}$.

- If the plant $P(s)$ is asymptotically stable and $C(s)$ is minimum phase, the optimization procedure is applied to the open-loop transfer function $P(s)$, with input $u^{*}$ and output $y^{*}$. Signal $r^{*}$ is then obtained by dynamic inversion on controller $C(s)$.

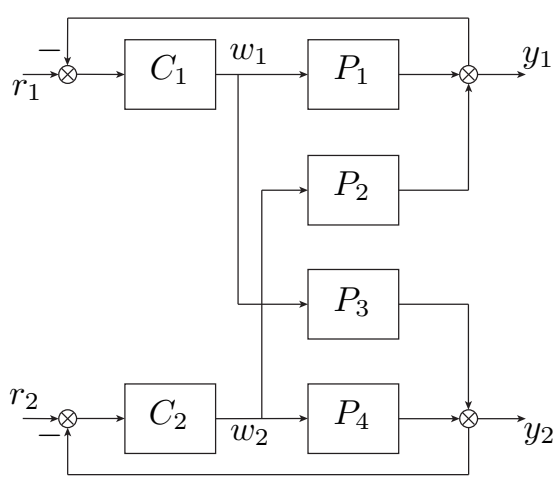

Fig. 3. The considered TITO control scheme.

The first procedure has the advantage that can be applied to any stabilizable system $P$. The second can be applied only to stable systems but has the advantage that is simpler computationally. In the following simulations the second procedure will be used.

Remark 3. The proposed approximation to determining the minimum-time feedforward action depends on the sampling time $T$. The smaller is $T$, the better is the approximation. A convergence result when $T \rightarrow 0$ has been given for SISO systems in [15]. Relying upon the discretization, the proposed method can be easily extended to MIMO plants with dead times (cf. examples in Section 3 ). In another direction, the method can also accommodate constraints on the time rate of the control variables (cf. example 1 in Section 3 and [20]).

\section{SIMULATION RESULTS}

The approach presented in Section 2.2 has been applied in simulation to two-inputs-two-outputs (TITO) systems with the matrix transfer function

$$
\mathbf{P}(s)=\left[P_{i j}(s)\right] \quad i, j=1,2 .
$$

The feedback regulation is obtained by means of decentralized output-filtered PID controllers in ideal form, namely,

where

$$
\mathbf{C}(s)=\left[\begin{array}{cc}
C_{1}(s) & 0 \\
0 & C_{2}(s)
\end{array}\right],
$$

$$
C_{j}(s)=K_{p j}\left(1+\frac{1}{T_{i j} s}+T_{d j} s\right) \frac{1}{T_{f j} s+1} \quad j=1,2 .
$$

The constants $K_{p j}, T_{i j}, T_{d j},(j=1,2)$ are, respectively, the proportional gain, the integral time constant and the derivative time constant of the PID controller that handles loop $j$. The output filter time constant $T_{f j}$ is selected such that the (high-frequency) measurement noise is filtered and, at the same time, the filter dynamics does not influence the control system dynamics. The TITO control scheme is outlined in Figure 3. The aim of the following simulations is to determine the reference signals $r_{1}$ and $r_{2}$, which permit to obtain a minimum-time step responses, while satisfying constraints on the maximum values of the control variables $w_{1}$ and $w_{2}$, and of the outputs $y_{1}$ and $y_{2}$.

First, a constrained minimum-time control problem has to be solved for the system described by transfer function $\mathbf{P}(s)$. Then, by a dynamic inversion procedure on the PID controller it is possible to obtain the required reference signals $r_{1}$ and $r_{2}$. Following the procedure presented in section 2.3, a numerical approximation of the generalized bang-bang signals $u_{1}$ and $u_{2}$ is determined by solving a Linear Programming Problem (LPP). In the following 


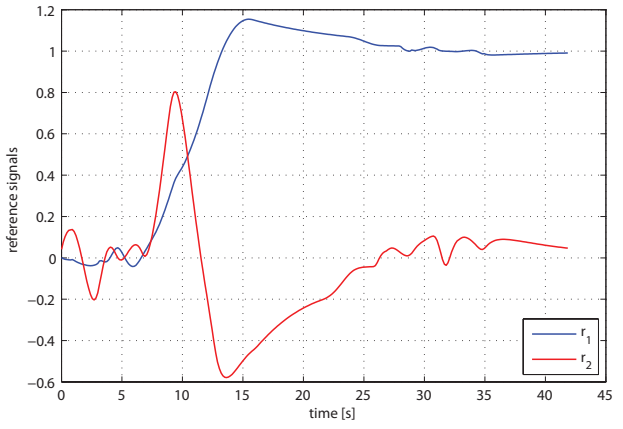

Fig. 4. Reference signals $r_{1}$ and $r_{2}$ for example 1 .

examples the LPP is solved by means of Yalmip, a toolbox for modeling and optimization in MATLAB ([21]).

\subsection{Example 1}

In the first example the distillation column model reported in $[22]$ is considered:

$$
\mathbf{P}(s)=\left[\begin{array}{cc}
\frac{12.8 e^{-s}}{1+16.7 s} & \frac{-18.9 e^{-3 s}}{1+21 s} \\
\frac{6.6 e^{-7 s}}{1+10.9 s} & \frac{-19.4 e^{-3 s}}{1+14.4 s}
\end{array}\right]
$$

The controllers are two output-filtered PID with the parameters tuned as follows:

$$
\begin{gathered}
K_{p 1}=0.61 \quad T_{i 1}=8.42 \quad T_{d 1}=0.26 \\
K_{p 2}=-0.12 \quad T_{i 2}=7.68 \quad T_{d 2}=0.73
\end{gathered}
$$

The filter time constant is selected in both cases as $T_{f}=0.1$. Since we impose a constraint also on the time derivatives of $w_{1}$ and $w_{2}$, in the optimization procedure we consider as input signal $u=\left[u_{1}, u_{2}\right]=\left[\frac{d w_{1}}{d t}, \frac{d w_{2}}{d t}\right]$ and as output $y=\left[w_{1}, w_{2}, y_{1}, y_{2}\right]$. The input and output signals satisfy conditions $u(t) \in \mathcal{U}$ and $y(t) \in \mathcal{Y}, \forall t \in \mathbb{R}$, where $\mathcal{U}$ and $\mathcal{Y}$ are, respectively, the input and the output constraints sets given by

$$
\begin{gathered}
\mathcal{U}=\left\{\left(u_{1}, u_{2}\right): U_{C}^{-} \leq u_{1}, u_{2} \leq U_{C}^{+}\right\}, \\
\mathcal{Y}=\left\{\left(w_{1}, w_{2}, y_{1}, y_{2}\right): W_{C}^{-} \leq w_{1}, w_{2} \leq W_{C}^{+}\right. \\
\left.\wedge Y_{C}^{-} \leq y_{1}, y_{2} \leq Y_{C}^{+}\right\} .
\end{gathered}
$$

The constraints on the outputs $y_{1}$ and $y_{2}$ are chosen to be $Y_{C}^{-}=-0.02$ and $Y_{C}^{+}=1.02$ which corresponds to allow maximum overshooting and undershooting of $2 \%$. The other constraints are set as follows: $W_{C}^{-}=-0.2$, $W_{C}^{+}=0.2, U_{C}^{-}=-0.01$ and $U_{C}^{+}=0.01$.

The sampling period is equal to $0.15 \mathrm{~s}$. The application of the proposed methodology provides the closed-loop command inputs $r_{1}$ and $r_{2}$ shown in Figure 4, the control variables plotted in Figure 5, the process variables plotted in Figure 6) and the first derivative of the control variables $u_{1}$ and $u_{2}$ shown in Figure 7 . In this case the optimal transition time is $35.04 \mathrm{~s}$. It can be noted that the posed constraints are not exceeded.

\subsection{Example 2}

As a second example, the following high-order process model is considered:

$\mathbf{P}(s)=\left[\begin{array}{c}\frac{1.2 e^{-3 s}}{(1+10 s)(1+5)^{2}} \\ \frac{0.6 e^{-10 s}}{(1+30 s)(1+20 s)(1+10 s)}\end{array}\right.$

$$
\frac{0.4 e^{-20 s}}{\frac{0.8 e^{-2 s}}{(1+60 s)(1+30 s)(1+10 s)}}
$$

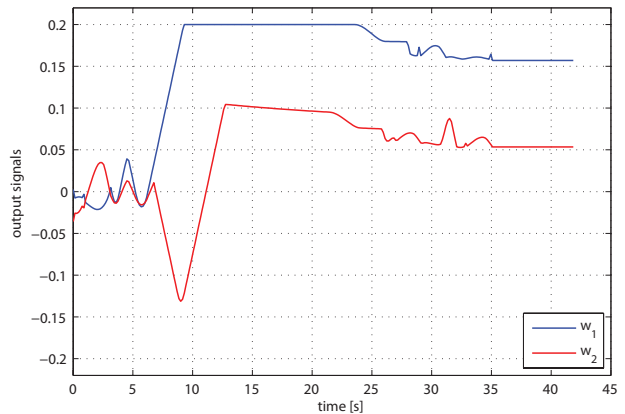

Fig. 5. Control variables $w_{1}$ and $w_{2}$ for example 1 .

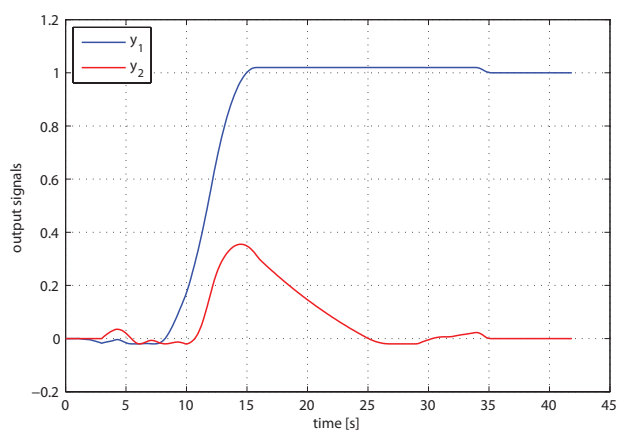

Fig. 6. Process variables $y_{1}$ and $y_{2}$ for example 1 .

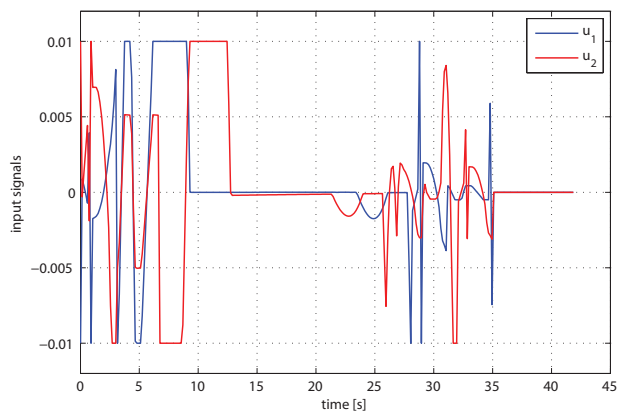

Fig. 7. First derivative of the control variables $u_{1}$ and $u_{2}$ for example 1 .

The controllers parameters are tuned as follows:

$$
\begin{array}{llll}
K_{p 1}=1.06 & T_{i 1}=14.4 & T_{d 1}=1.59 & T_{f 1}=0.002 \\
K_{p 2}=1.78 & T_{i 2}=6.09 & T_{d 2}=0.62 & T_{f 1}=0.002
\end{array}
$$

We want to obtain a rest-to-rest transition from 0 to 1 for both the outputs $y_{1}$ and $y_{2}$. In this second example the control inputs for the minimum-time optimization problem are the actual system inputs $u_{1}=w_{1}$ and $u_{2}=w_{2}$, while the considered output is $y=\left[y_{1}, y_{2}\right]$, with $u(t) \in \mathcal{U}$ and $y(t) \in \mathcal{Y}, t \in\left[0, t_{f}\right]$, where $\mathcal{U}$ and $\mathcal{Y}$ are, respectively, the input and the output constraints sets given by

$$
\begin{gathered}
\mathcal{U}=\left\{u_{1}, u_{2}: U_{C}^{-} \leq u_{1}(t), u_{2}(t) \leq U_{C}^{+}\right\}, \\
\mathcal{Y}=\left\{y_{1}, y_{2}: Y_{C}^{-} \leq y_{1}(t), y_{2}(t) \leq Y_{C}^{+}\right\} .
\end{gathered}
$$

The constraints on the outputs $y_{1}$ and $y_{2}$ are chosen to be $Y_{C}^{-}=-0.02$ and $Y_{C}^{+}=1.02$ which corresponds to allow maximum overshooting and undershooting of $2 \%$, while the input constraints are set to be $U_{C}^{-}=-10$ and $U_{C}^{+}=10$. The discretization period is equal to $0.05 \mathrm{~s}$.

The application of the proposed methodology provides the closed-loop command inputs $r_{1}$ and $r_{2}$ shown in Figure 8, the process variables of Figure 9 and the control variables 


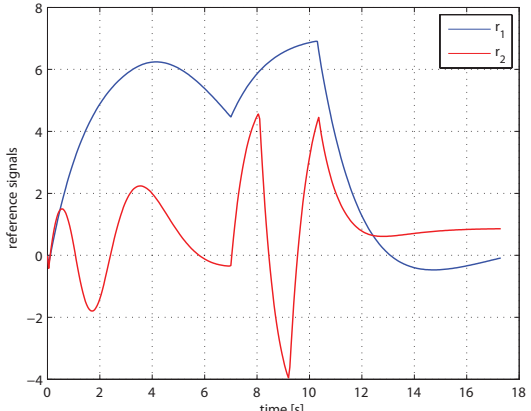

Fig. 8. Command signals $r_{1}$ and $r_{2}$ for example 2 .

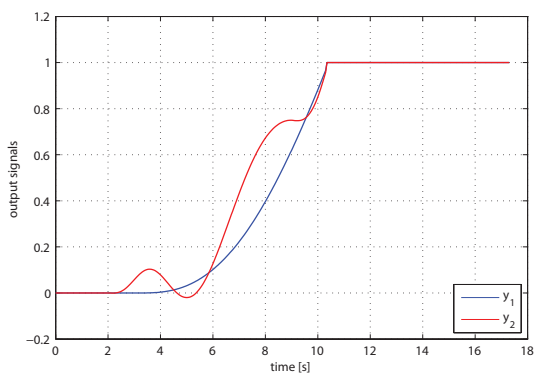

Fig. 9. Output signals $y_{1}$ and $y_{2}$ for example 2 .

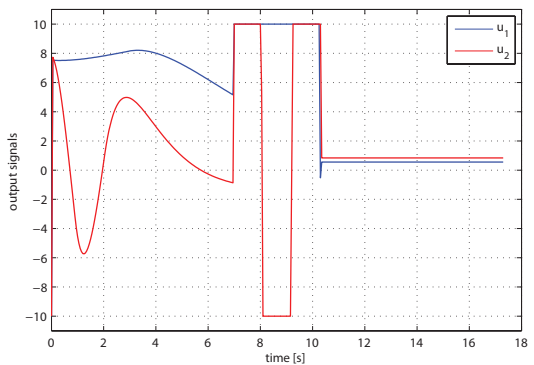

Fig. 10. Control variables $u_{1}$ and $u_{2}$ for example 2 .

$w_{1}$ and $w_{2}$ shown in Figure 10. In this case the optimal transition time is $10.30 \mathrm{~s}$.

The comparison of the obtained results with the ones presented in [17] shows that the use of a feedforward action in the set-point signals $r$ can improve significantly the performance of a PID controller.

\section{CONCLUSIONS}

The paper has considered the set-point regulation of multivariable plants subject to input and output amplitude constraints. The proposed approach uses decentralized PID feedback control and a feedforward action to achieve restto-rest minimum-time output transitions.

Future work will include the determination of suitable tuning rules to be combined to the minimum-time restto-rest feedforward control law (see [18]) and the design of set-point filters for an easier implementation of the methodology (see [19]).

\section{REFERENCES}

[1] A. O'Dwyer, Handbook of PI and PID controller tuning rules, Imperial College Press, 2006.

[2] J. Dong, C.B. Brosilow, "Design of robust multivariable PID controller via IMC", Proceedings American Control Conference, pp. 3380-3384, 1997.
[3] M. Lee, K. Lee, C. Kim, J. Lee, "Analytical design of multiloop PID controllers for desired closed-loop responses", AIChE Journal, Vol. 50, No. 7, pp. 1631$1635,2004$.

[4] K. J. Åström, T. Hägglund, Advanced PID control, ISA Press, Research Triangle Park (NC), 2006.

[5] A. Visioli, Practical PID Control, Springer, London (UK), 2006.

[6] C.-C. Hang, L. Cao, "Improvement of transient response by means of variable set-point weighting", IEEE Transactions on Industrial Electronics, Vol. 43, No. 4, pp. 477-484, 1996.

[7] A. Visioli, "Fuzzy logic based set-point weight tuning of PID controllers", IEEE Transactions on Systems, Man, and Cybernetics - Part A, Vol. 29, No. 6, pp. 587-592, 1999.

[8] A. Wallen, Tools for autonomous process control, $\mathrm{PhD}$ thesis, Lund Institute of Technology, 2000.

[9] A. Wallen, K. J. Åström, "Pulse-step control", Preprints of the 15th IFAC World Congress on Automatic Control, Barcelona (E), 2002.

[10] A. Piazzi, A. Visioli, "A noncausal approach for PID control", Journal of Process Control, Vol. 16, No. 8, pp. 831-843, 2006.

[11] A. Piazzi, A. Visioli, "Pareto optimal feedforward constrained regulation of MIMO linear systems", Preprints 16th IFAC World Congress on Automatic Control, Prague (CZ), July 2005.

[12] S. Piccagli, A. Visioli, "Minimum-time feedforward plus PID control for MIMO systems", Preprints 17th IFAC World Congress on Automatic Control, Seoul (ROK), pp. 12917-12922, July 2008.

[13] A.H. Glattfelder, W. Schaufelberger, "Control Systems with Input and Output Constraints", Springer, London (UK), 2003.

[14] L. Consolini, A. Piazzi, A. Visioli, "Minimum-time feedforward control for industrial processes", Proceedings of the 2007 European Control Conference, Kos (Greece), pp. 5282-5287, 2007.

[15] L. Consolini, A. Piazzi, "Generalized bang-bang control for feedforward constrained regulation", Automatica, Vol. 45, No. 10, pp. 2234-2243, 2009.

[16] J. W. Polderman, J. C. Willems, Introduction to mathematical system theory, Springer, New York, NY, 1998.

[17] M. Veronesi, A. Visioli, "An Automatic Tuning Method for Multiloop PID Controllers", Preprints of the 18th IFAC World Congress, Milan (Italy), 2011.

[18] S. Piccagli, A. Visioli, "PID tuning for minimum-time rest-to-rest transitions", Preprints 18th IFAC World Congress, Milan (I), pp. 5771-5776, August-September 2011.

[19] M. Beschi, A. Piazzi, A. Visioli, "On the practical implementation of a noncausal feedforward technique for PID control", Proceedings European Control Conference, Budapest (HU), pp. 1806-1811, August 2009.

[20] F. Mesquine, F. Tadeob, A. Benzaouia, "Regulator problem for linear systems with constraints on control and its increment or rate", Automatica, Vol. 40, pp. 1387-1395, 2004.

[21] J. Löfberg, "A Toolbox for Modeling and Optimization in MATLAB", Proceedings of the CACSD Conference, Taipei, Taiwan, 2004.

[22] R. K. Wood, M. W. Berry, "Terminal composition control of a binary distillation column", Chemical Engineering Science, No. 16, Vol. 28, pp.1707-1710, 1973. 